\title{
FFLP problem with symmetric trapezoidal fuzzy numbers
}

\author{
Reza Daneshrad* and Davood Jafari
}

Department of Industrial Engineering, Parand Branch, Islamic Azad University, Parand, Iran

\begin{tabular}{l}
\hline C H R O N I C L E \\
\hline Article history: \\
Received December 10, 2014 \\
Received in revised format: \\
January 4, 2015 \\
Accepted January 5, 2015 \\
Available online \\
January 5 2015 \\
\hline Keywords: \\
Fully fuzzy linear programming \\
Fuzzy primal simplex algorithm \\
Ranking \\
Symmetric trapezoidal fuzzy \\
number
\end{tabular}

\begin{abstract}
The most popular approach for solving fully fuzzy linear programming (FFLP) problems is to convert them into the corresponding deterministic linear programs. Khan et al. (2013) [Khan, I. U., Ahmad, T., \& Maan, N. (2013). A simplified novel technique for solving fully fuzzy linear programming problems. Journal of Optimization Theory and Applications, 159(2), 536546.] claimed that there had been no method in the literature to find the fuzzy optimal solution of a FFLP problem without converting it into crisp linear programming problem, and proposed a technique for the same. Others showed that the fuzzy arithmetic operation used by Khan et al. (2013) had some problems in subtraction and division operations, which could lead to misleading results. Recently, Ezzati et al. (2014) [Ezzati, R., Khorram, E., \& Enayati, R. (2014). A particular simplex algorithm to solve fuzzy lexicographic multi-objective linear programming problems and their sensitivity analysis on the priority of the fuzzy objective functions. Journal of Intelligent and Fuzzy Systems, 26(5), 2333-2358.] defined a new operation on symmetric trapezoidal fuzzy numbers and proposed a new algorithm to find directly a lexicographic/preemptive fuzzy optimal solution of a fuzzy lexicographic multiobjective linear programming problem by using new fuzzy arithmetic operations, but their model was not fully fuzzy optimization. In this paper, a new method, by using Ezzati et al. (2014)'s fuzzy arithmetic operation and a fuzzy version of simplex algorithm, is proposed for solving FFLP problem whose parameters are represented by symmetric trapezoidal fuzzy number without converting the given problem into crisp equivalent problem. By using the proposed method, the fuzzy optimal solution of FFLP problem can be easily obtained. A numerical example is provided to illustrate the proposed method.
\end{abstract}

(C) 2015 Growing Science Ltd. All rights reserved.

\section{Introduction}

Linear programming (LP) is one of the most important operation research techniques applied to solve many decision making problems. Any LP model representing real-world situations involves various parameters, whose values are assigned by experts. However, both decision maker and experts frequently do not know the value of the parameters, precisely. Therefore, it is useful to consider the knowledge of experts about the parameters as fuzzy data. The main objective in fuzzy linear programming (FLP) is to find the best possible solution with imprecise, vague, uncertain or incomplete information.

\footnotetext{
* Corresponding author.

E-mail address: daneshrad.reza@gmail.com (R. Daneshrad)

C 2015 Growing Science Ltd. All rights reserved.

doi: $10.5267 /$ j.ds1.2015.1.004
} 
The concept of a fuzzy decision process, as an intersection of the fuzzy objective function and resource constraints, was first introduced by Bellman and Zadeh (1970). From this idea, Tanaka et al. (1974) and Zimmermann (1978) introduced LP problems in a fuzzy environment. The FLP in which all the parameters as well as variables are represented by fuzzy numbers in known as fully fuzzy linear programing problem. Buckley and Feuring (2000) introduced a method to find a solution for FFLP problem by changing the objective function into the multiobjective LP problems. Dehghan et al. (2006) used a computational method to find the exact solution of a FFLP problem. Hashemi et al. (2006) introduced the weak duality theorem based on alphabetic order function for FFLP problem. Allahviranloo et al. (2008) proposed a method for solving FFLP problem by using a kind of defuzzification method.

Hosseinzadeh Lotfi et al. (2009) discussed FFLP problem where all parameters and variables were symmetric triangular fuzzy numbers. Kumar et al. (2010) proposed a method to find the fuzzy optimal solution of FFLP problem with equality constraints. Nasseri et al. (2013) proposed a new method for finding the fuzzy solution of FFLP problems with inequality constraints. Hatami and Kazemipoor (2014) proposed a new method for finding the fuzzy solution of the FFLP problem by converting it into LP problem. Khan et al. (2013) claimed there is no method in the literature to find the fuzzy optimal solution of a FFLP problem without converting it into crisp linear programming problem, and proposed a technique for the same. Bhardwaj and Kumar (2013) showed that according to fuzzy arithmetic operation used by Khan et al. (2013) for the fuzzy number $\tilde{A}$ the properties $\frac{\tilde{A}}{\tilde{A}} \approx \tilde{1}$ and $\tilde{A}-\tilde{A} \approx \tilde{0}$ could not be satisfied, there are errors in the proposed method by Khan et al. (2013), and there is no other option to solve the FFLP problems without converting them into crisp linear programming problems.

Recently, Ezzati et al. (2014) defined a new operation on symmetric trapezoidal fuzzy numbers that the properties $\frac{\tilde{A}}{\tilde{A}} \approx \tilde{1}$ and $\tilde{A}-\tilde{A} \approx \tilde{0}$ are satisfied and based on new fuzzy arithmetic operations proposed, a new algorithm to find directly a lexicographic/preemptive fuzzy optimal solution of a fuzzy lexicographic multi-objective linear programming problem was proposed, but their model was not fully fuzzy optimization. In this paper, a new method by using Ezzati et al. (2014)'s fuzzy arithmetic operation and a fuzzy version of simplex algorithm is proposed for solving FFLP problem whose parameters all are represented by symmetric trapezoidal fuzzy number without converting the given problem into crisp equivalent problem. By using the proposed method the fuzzy optimal solution of FFLP problem can be easily obtained.

This paper is organized as follows: In section 2 some basic definitions and arithmetic of symmetric trapezoidal fuzzy numbers and a review of formulation of FFLP problem are presented. In section 3 a new method is proposed for FFLP problem and in section 4 a numerical example is solved. Finally conclusions are discussed in section 5.

\section{Preliminaries}

In this section, some basic definitions and arithmetic operations of symmetric trapezoidal fuzzy numbers are reviewed (Ezzati et al., 2014). In addition, a brief review of using ranking function and FFLP problem is given (Ganesan \& Veeramani, 2006).

Definition 2.1 Let $X$ be a universal set. Then a fuzzy subset $\tilde{A}$ of $X$ is defined by its membership function $\mu_{\tilde{A}}: X \rightarrow[0,1]$ which assign a real number $\mu_{\tilde{A}(x)}$ in the interval $[0,1]$, to each element $\mathrm{x} \in \mathrm{X}$ where the value of $\mu_{\tilde{A}(x)}$ at $x$ shows the grade of membership $x$ in $\tilde{A}$.

Definition 2.2 A fuzzy number $\tilde{A}=\left(a^{L}, a^{U}, \alpha, \alpha\right)$ is said to be a symmetric trapezoidal fuzzy number if its membership function is given as follows, 


$$
\mu_{\tilde{A}(x)}=\left\{\begin{array}{cc}
\frac{x-\left(a^{L}-\alpha\right)}{\alpha}, & a^{L}-\alpha \leq x \leq a^{L} \\
1, & a^{L} \leq x \leq a^{U} \\
\frac{\left(a^{U}+\alpha\right)-x}{\alpha}, & a^{U} \leq x \leq a^{U}+\alpha \\
0, & \text { else }
\end{array}\right.
$$

Definition 2.3 Let $\tilde{A}=\left(a^{L}, a^{U}, \alpha, \alpha\right)$ and $\tilde{B}=\left(b^{L}, b^{U}, \beta, \beta\right)$ be two trapezoidal fuzzy numbers then:

(i) $\tilde{A}+\tilde{B}=\left(a^{L}+b^{L}, a^{U}+b^{U}, \alpha+\beta, \alpha+\beta\right)$

(ii) $\quad \tilde{A}-\tilde{B}=\left(a^{L}-b^{U}, a^{U}-b^{L}, \alpha+\beta, \alpha+\beta\right)$

(iii) $\tilde{A} \times \tilde{B}=\left(\frac{a^{L}+a^{U}}{2} \times \frac{b^{L}+b^{U}}{2}-w, \frac{a^{L}+a^{U}}{2} \times \frac{b^{L}+b^{U}}{2}+w,\left|w-w^{\prime}\right|,\left|w-w^{\prime}\right|\right)$

where $w=\min \left(\left[\frac{a^{L}+a^{U}}{2}\right]\left[\frac{b^{L}+b^{U}}{2}\right]-\min (m), \max (m)-\left[\frac{a^{L}+a^{U}}{2}\right]\left[\frac{b^{L}+b^{U}}{2}\right]\right)$

$w^{\prime}=\min \left(\left[\frac{a^{L}+a^{U}}{2}\right]\left[\frac{b^{L}+b^{U}}{2}\right]-\min (n), \max (n)-\left[\frac{a^{L}+a^{U}}{2}\right]\left[\frac{b^{L}+b^{U}}{2}\right]\right)$

and $m=\left(a^{L} b^{L}, a^{L} b^{U}, a^{U} b^{L}, a^{U} b^{U}\right)$,

$n=\left(\left(a^{L}-\alpha\right)\left(b^{L}-\beta\right),\left(a^{L}-\alpha\right)\left(b^{U}+\beta\right),\left(a^{U}+\alpha\right)\left(b^{L}-\beta\right),\left(a^{U}+\alpha\right)\left(b^{U}+\beta\right)\right)$.

Let $\tilde{A}=\left(a^{L}, a^{U}, \alpha, \alpha\right)$ and $\tilde{B}=\left(b^{L}, b^{U}, \beta, \beta\right)$ be two trapezoidal fuzzy numbers and let $0 \notin\left[b^{L}-\right.$ $\left.\beta, b^{U}+\beta\right]$. Define,

(iv) $\quad \tilde{A} \div \tilde{B}=\left(\frac{a^{L}+a^{U}}{b^{L}+b^{U}}-w, \frac{a^{L}+a^{U}}{b^{L}+b^{U}}+w,\left|w-w^{\prime}\right|,\left|w-w^{\prime}\right|\right)$

where $w=\min \left\{\frac{a^{L}+a^{U}}{b^{L}+b^{U}}-\min (m), \max (m)-\frac{a^{L}+a^{U}}{b^{L}+b^{U}}\right\}, w^{\prime}=\min \left\{\frac{a^{L}+a^{U}}{b^{L}+b^{U}}-\min (n), \max (n)-\right.$ $\left.\frac{a^{L}+a^{U}}{b^{L}+b^{U}}\right\}, m=\left(\frac{a^{L}}{b^{L}}, \frac{a^{L}}{b^{U}}, \frac{a^{U}}{b^{L}}, \frac{a^{U}}{b^{U}}\right), n=\left(\frac{a^{L}-\alpha}{b^{L}-\beta}, \frac{a^{L}-\alpha}{b^{U}+\beta}, \frac{a^{U}+\alpha}{b^{L}-\beta}, \frac{a^{U}+\alpha}{b^{U}+\beta}\right)$

Definition 2.4 For any symmetric trapezoidal fuzzy number $\tilde{A}$, we define $\tilde{A} \geqslant \tilde{0}$ if there exist $c, \delta, \geq 0$ such that $\tilde{A} \geqslant(-c, c, \delta, \delta)$. We also denote $(-c, c, \delta, \delta)$ by $\tilde{0}$.

Definition 2.5 For any symmetric trapezoidal fuzzy number $\tilde{A}$, we define $\tilde{A} \geqslant \tilde{1}$ if there exist $c, \delta \geq 0$ such that $\tilde{A} \geqslant(-c+1, c+1, \delta, \delta)$. We also denote $(-c+1, c+1, \delta, \delta)$ by $\tilde{1}$.

Definition 2.6 A ranking function is a function $\mathrm{R}: \mathrm{F}(\mathrm{X}) \rightarrow \mathrm{X}$ which maps each fuzzy number into the real line, where a natural order exists. Let $\tilde{A}=\left(a^{L}, a^{U}, \alpha, \alpha\right)$ be any symmetric trapezoidal fuzzy number, then

$$
R(\tilde{A})=\frac{a^{L}+a^{U}}{2}
$$

Definition 2.7 Let $\tilde{A}=\left(a^{L}, a^{U}, \alpha, \alpha\right)$ and $\tilde{B}=\left(b^{L}, b^{U}, \beta, \beta\right)$ be two symmetric trapezoidal fuzzy numbers then:

(i) $\quad \tilde{A} \leq \tilde{B}$ iff $R(\tilde{A}) \leq R(\tilde{B})$

(ii) $\quad \tilde{A}<\tilde{B}$ iff $R(\tilde{A})<R(\tilde{B})$

Definition 2.8 A FFLP problem is defined as follows:

$\max \tilde{Z} \approx \tilde{c} \tilde{x}$ 
$\tilde{x} \geqslant \tilde{0}$

where $\tilde{A}=\left(\tilde{a}_{i j}\right)_{m \times n}, \tilde{c}=\left(\tilde{c}_{j}\right)_{1 \times n}, \quad \tilde{b}=\left(\tilde{b}_{i}\right)_{m \times 1}, \quad \tilde{x}=\left(\tilde{x}_{j}\right)_{1 \times n} \quad$ and $\quad \tilde{a}_{i j}, \tilde{b}_{i}, \tilde{c}_{j}, \tilde{x}_{j} \in F(X)(i=$ $1,2, \ldots, m, j=1,2, \ldots, n), \tilde{b}_{i}, \tilde{x}_{j} \geqslant \tilde{0}$.

Definition 2.9 Let the i-th fuzzy constraint of the FFLP problem be $\sum_{j=1}^{n} \tilde{a}_{i j} \tilde{x}_{j} \preccurlyeq($ or $\geqslant) \tilde{b}_{i}$ where $\tilde{b}_{i} \geqslant$ $\tilde{0}$ then a fuzzy variable $\tilde{s}_{i} \geqslant \tilde{0}$ and $\sum_{j=1}^{n} \tilde{a}_{i j} \tilde{x}_{j}+\tilde{s}_{i}\left(\right.$ or $\left.-\tilde{s}_{i}\right) \approx \tilde{b}_{i}$ is called a fuzzy slack (or surplus) variable.

Definition 2.10 Given a system of $\mathrm{m}$ fuzzy linear equation involving trapezoidal fuzzy numbers in $n$ unknowns $(n \geq m), \tilde{A} \tilde{x} \approx \tilde{b}$, where $\tilde{A}$ is a $m \times n$ matrix and $\operatorname{rank}$ of $\tilde{A}$ is $m$. Let the columns of $\tilde{A}$ corresponding of fuzzy variables $\tilde{x}_{k_{1}}, \ldots, \tilde{x}_{k_{m}}$ are linearly independent then $\tilde{x}_{k_{1}}, \ldots, \tilde{x}_{k_{m}}$ are said to be fuzzy basic variables and remaining $(n-m)$ variables are called fuzzy nonbasic variables. Let $\tilde{B}$ the basis matrix formed by linearly independent columns of $\tilde{A}$. The value of $\tilde{X}_{B}=\left(\tilde{x}_{k_{1}}, \ldots, \tilde{x}_{k_{m}}\right)$ is obtained by using $\tilde{X}_{B}=\widetilde{B}^{-1} \tilde{b}$, where $k_{j} \in\{1,2, \ldots, n\}, k_{i} \neq k_{j}, i, j=1,2, \ldots, m$ and the values of nonbasic variables are assumed to be zero. The combined solution formed by using the values of fuzzy basic variables and fuzzy nonbasic variables are called fuzzy basic solution. See in (Ganesan \& Veeramani, 2006).

Definition 2.11 Any $\tilde{X}$ which satisfies all the constraints $\tilde{A} \tilde{x} \approx \tilde{b}$ and nonnegative restricted $\tilde{x} \geqslant \tilde{0}$ of Eq. (3) is said to be a fuzzy feasible solution of Eq. (3).

Definition 2.12 Let $Q$ be the set of all fuzzy feasible solution of Eq. (3). A fuzzy feasible solution $\tilde{X}_{0} \in$ $Q$ is said to be a fuzzy optimal solution of (3) if $\tilde{c} \tilde{X}_{0} \geqslant \tilde{c} \tilde{X}$.

\section{Proposed method}

In this section, the steps of the fuzzy simplex algorithm is proposed to obtain the fuzzy optimal solution for FFLP problem as follows:

Step 1: convert all the inequalities of the constraints into equation by introducing fuzzy slack or surplus variables. (Put the coefficient of these fuzzy slack and surplus variables equal to zero in the objective function) and suppose an initial fuzzy basic feasible solution with basis $\tilde{B}$ is at hand.

Steps 2: construct the fuzzy simplex table in the following format:

Table 1

Fuzzy simplex Table

\begin{tabular}{ccccccc}
\hline$\tilde{X}_{B}$ & $\tilde{x}_{1}$ & $\ldots$ & $\tilde{x}_{r}$ & $\ldots$ & $\tilde{x}_{n}$ & RHS \\
\hline$\tilde{z}$ & $\tilde{z}_{1}-\tilde{c}_{1}$ & $\ldots$ & $\tilde{z}_{r}-\tilde{c}_{r}$ & $\ldots$ & $\tilde{z}_{n}-\tilde{c}_{n}$ & - \\
$\tilde{x}_{B 1}$ & $\tilde{a}_{11}$ & $\ldots$ & $\tilde{a}_{1 r}$ & $\ldots$ & $\tilde{a}_{1 n}$ & $\tilde{b}_{1}$ \\
$\vdots$ & $\vdots$ & & $\vdots$ & & $\vdots$ & $\vdots$ \\
$\tilde{x}_{B i}$ & $\tilde{a}_{i 1}$ & $\ldots$ & $\tilde{a}_{i r}$ & $\ldots$ & $\tilde{a}_{i n}$ & $\tilde{\bar{b}}_{r}$ \\
$\vdots$ & $\vdots$ & & $\vdots$ & & $\vdots$ & $\vdots$ \\
$\tilde{x}_{B m}$ & $\tilde{a}_{m 1}$ & $\ldots$ & $\tilde{a}_{m r}$ & $\ldots$ & $\tilde{a}_{m n}$ & $\tilde{b}_{m}$ \\
\hline
\end{tabular}

Step 3: find $\tilde{z}_{j}-\tilde{c}_{j}=\sum_{i=1}^{m} \tilde{c}_{B_{i}} \tilde{a}_{i j}-\tilde{c}_{j}, \forall j=1,2, \ldots, n$ 
(i) If $\tilde{z}_{j}-\tilde{c}_{j} \geqslant \tilde{0}, \forall j$ then the fuzzy basic feasible solution, obtained by using values of $\tilde{x}_{B_{1}}, \ldots, \tilde{x}_{B_{m}}$ of Table 1 , is fuzzy optimal solution and $\tilde{Z}$ is the optimal value of maximization problem.

(ii) If there exists any $j$ such that $\tilde{z}_{j}-\tilde{c}_{j} \preccurlyeq \tilde{0}$ proceed on to the next step.

Step 4: if there exists one or more fuzzy variables with $\tilde{z}_{j}-\tilde{c}_{j} \preccurlyeq \tilde{0}$ then the fuzzy variable corresponding to which the rank of $\tilde{z}_{j}-\tilde{c}_{j}$ is most negative will enter the basis. Let it be $\tilde{z}_{r}-\tilde{c}_{r}$ for some $j=r$.

(i) If $\tilde{a}_{i j} \preccurlyeq \tilde{0}, \forall i$ then there exist fuzzy unbounded solution to the given FFLP problem.

(ii) If $\tilde{a}_{i j} \geqslant \tilde{0}$ for one or more values of $i$ then compute

$$
\operatorname{minimum}\left\{R\left(\frac{\tilde{l}_{i}}{\tilde{a}_{i r}}\right), \tilde{a}_{i r}>\tilde{0}, i=1,2, \ldots, m\right\}
$$

where, $\tilde{l}_{i}$ is the value of $i$-th fuzzy basic variable. The fuzzy variable, corresponding to which minimum occurs, will leave the basis. Let the minimum occurs corresponding to $\tilde{x}_{B_{k}}$ then the common element $\tilde{a}_{k r}$, which occurs at intersection of k-th row and r-th column is known as the leading element.

Step 5: construct the new fuzzy simplex table and calculate the new entries for the fuzzy simplex table as follows:

$\hat{a}_{k j}=\frac{\tilde{a}_{k j}}{\tilde{a}_{k r}}$ and $\hat{a}_{i j}=\tilde{a}_{i j}-\frac{\tilde{a}_{k j}}{\tilde{a}_{k r}} \tilde{a}_{i r}$ where $i=1,2, \ldots, m, i \neq k, j=1,2, \ldots, n$.

Step 6: Repeat the computational procedure from step 3 to step 5 until either $(i)$ of step 3 or $(i)$ of step 4 is satisfied.

Step 7: if $\tilde{z}_{j}-\tilde{c}_{j} \approx \tilde{0}$ corresponding to any fuzzy nonbasic variable in the fuzzy optimal table (simplex table for which $\tilde{z}_{j}-\tilde{c}_{j} \geqslant \tilde{0}, \forall j$ ) then a fuzzy alternative solution may exist and to find it enter that fuzzy nonbasic variable into the basis and repeat once step 3 to. The obtained fuzzy optimal solution will be a fuzzy alternative optimal solution.

\section{A numerical example}

For an illustration of the proposed method, a FFLP problem is solved. Consider the following problem:

$\max \tilde{z} \approx(4,6,3,3) \tilde{x}_{1}+(2,4,1,1) \tilde{x}_{2}$

subject to

$$
\begin{aligned}
& (1,1,0,0) \tilde{x}_{1} \preccurlyeq(3,9,2,2) \\
& (1,1,0,0) \tilde{x}_{2} \preccurlyeq(5,11,3,3) \\
& (2,2,1,1) \tilde{x}_{1}+(2,4,1,1) \tilde{x}_{2} \preccurlyeq(15,33,4,4) \\
& \tilde{x}_{1}, \tilde{x}_{2} \geqslant \tilde{0}
\end{aligned}
$$

Using step 1 of the proposed algorithm, the formulated problem may be written as:

$$
\begin{aligned}
& \max \tilde{z} \approx \\
& \text { subject to } \\
& \qquad \begin{array}{l}
(1,6,3,3) \tilde{x}_{1}+(2,4,1,1) \tilde{x}_{2} \\
(1,1,0,0) \tilde{x}_{1}+(1,1,0,0) \tilde{x}_{3} \approx(3,9,2,2) \\
(2,2,1,1) \tilde{x}_{1}+(2,4,1,1) \tilde{x}_{2}+(1,1,0,0) \tilde{x}_{5} \approx(15,33,4,4)
\end{array}
\end{aligned}
$$




$$
\tilde{x}_{1}, \tilde{x}_{2}, \tilde{x}_{3}, \tilde{x}_{4}, \tilde{x}_{5} \geqslant \tilde{0}
$$

where $\tilde{x}_{3}, \tilde{x}_{4}, \tilde{x}_{5}$ are fuzzy slack variables. Using step 2 the fuzzy simplex table for the above problem is as follows,

\section{Table 2}

The initial fuzzy simplex tableau

\begin{tabular}{ccccccc}
\hline$\tilde{X}_{B}$ & $\tilde{x}_{1}$ & $\tilde{x}_{2}$ & $\tilde{x}_{3}$ & $\tilde{x}_{4}$ & $\tilde{x}_{5}$ & RHS \\
\hline$\tilde{z}$ & $(-6,-4,3,3)$ & $(-4,-2,1,1)$ & $\tilde{0}$ & $\tilde{0}$ & $\tilde{0}$ & $\tilde{0}$ \\
$\tilde{x}_{3}$ & $(1,1,0,0)$ & $\tilde{0}$ & $(1,1,0,0)$ & $\tilde{0}$ & $\tilde{0}$ & $(3,9,2,2)$ \\
$\tilde{x}_{4}$ & $\tilde{0}$ & $(1,1,0,0)$ & $\tilde{0}$ & $(1,1,0,0)$ & $\tilde{0}$ & $(5,11,3,3)$ \\
$\tilde{x}_{5}$ & $(2,2,1,1)$ & $(2,4,1,1)$ & $\tilde{0}$ & $\tilde{0}$ & $(1,1,0,0)$ & $(15,33,4,4)$ \\
\hline
\end{tabular}

Using step 4 of the proposed algorithm, fuzzy variable $\tilde{x}_{1}$ will enter the basis and the fuzzy variable $\tilde{x}_{3}$ will leave the basis. Using step 5 of the proposed algorithm new fuzzy simplex table is:

Table 3

The first iteration

\begin{tabular}{ccccccc}
\hline$\tilde{X}_{B}$ & $\tilde{x}_{1}$ & $\tilde{x}_{2}$ & $\tilde{x}_{3}$ & $\tilde{x}_{4}$ & $\tilde{x}_{5}$ & RHS \\
\hline$\tilde{z}$ & $(-2,2,6,6)=\tilde{0}$ & $(-4,-2,1,1)$ & $(4,6,3,3)$ & $\tilde{0}$ & $\tilde{0}$ & $(12,48,11,11)$ \\
$\tilde{x}_{1}$ & $(1,1,0,0)=\tilde{1}$ & $\tilde{0}$ & $(1,1,0,0)$ & $\tilde{0}$ & $\tilde{0}$ & $(3,9,2,2)$ \\
$\tilde{x}_{4}$ & $\tilde{0}$ & $(1,1,0,0)$ & $\tilde{0}$ & $(1,1,0,0)$ & $\tilde{0}$ & $(5,11,3,3)$ \\
$\tilde{x}_{5}$ & $(0,0,2,2)=\tilde{0}$ & $(2,4,1,1)$ & $(-2,-2,1,1)$ & $\tilde{0}$ & $(1,1,0,0)$ & $(-3,27,9,9)$ \\
\hline
\end{tabular}

According to Table 3 , fuzzy variable $\tilde{x}_{2}$ will enter the basis and the fuzzy variable $\tilde{x}_{5}$ will leave the basis. Using step 5 of the proposed algorithm, the new fuzzy simplex table is as follows,

Table 4

The optimal solution

\begin{tabular}{ccccccc}
\hline$\tilde{X}_{B}$ & $\tilde{x}_{1}$ & $\tilde{x}_{2}$ & $\tilde{x}_{3}$ & $\tilde{x}_{4}$ & $\tilde{x}_{5}$ & RHS \\
\hline$\tilde{z}$ & $(-2,2,16,16)=\tilde{0}$ & $\left(-3,3, \frac{9}{5}, \frac{9}{5}\right)=\tilde{0}$ & $\left(1,5, \frac{19}{5}, \frac{19}{5}\right)$ & $\tilde{0}$ & $\left(\frac{1}{2}, \frac{3}{2}, \frac{3}{10}, \frac{3}{10}\right)$ & $(6,78,65,65)$ \\
$\tilde{x}_{1}$ & $(1,1,0,0)=\tilde{1}$ & $\tilde{0}$ & $(1,1,0,0)$ & $\tilde{0}$ & $\tilde{0}$ & $(3,9,2,2)$ \\
$\tilde{x}_{4}$ & $(0,0,2,2)=\tilde{0}$ & $\left(\frac{-1}{2}, \frac{1}{2}, \frac{3}{10}, \frac{3}{10}\right)=\tilde{0}$ & $\left(\frac{3}{6}, \frac{5}{6}, \frac{3}{10}, \frac{3}{10}\right)$ & $(1,1,0,0)$ & $\left(\frac{-5}{12}, \frac{-3}{12}, \frac{1}{20}, \frac{1}{20}\right)$ & $\left(\frac{-9}{2}, \frac{25}{2}, \frac{27}{2}, \frac{27}{2}\right)$ \\
$\tilde{x}_{2}$ & $(0,0,2,2)=\tilde{0}$ & $\left(\frac{1}{2}, \frac{3}{2}, \frac{3}{10}, \frac{3}{10}\right)=\tilde{1}$ & $\left(\frac{-5}{6}, \frac{-3}{6}, \frac{3}{10}, \frac{3}{10}\right)$ & $\tilde{0}$ & $\left(\frac{3}{12}, \frac{5}{12}, \frac{1}{20}, \frac{1}{20}\right)$ & $\left(\frac{-3}{2}, \frac{19}{2}, \frac{21}{2}, \frac{21}{2}\right)$ \\
\hline
\end{tabular}

Therefore, the fuzzy optimal solution of the FFLP problem which is obtained by the fuzzy simplex algorithm is $\tilde{x}_{1}=(3,9,2,2), \tilde{x}_{2}=\left(\frac{-3}{2}, \frac{19}{2}, \frac{21}{2}, \frac{21}{2}\right)$ and the fuzzy optimal value of its objective function is $\tilde{z}=(6,78,65,65)$. 


\section{Conclusion}

Since, in standard fuzzy arithmetic operations we have some problems in division and subtraction operations so there is no other option to solve fully fuzzy linear programming problems without converting them into the corresponding deterministic linear programs. In this paper a new method by using Ezzati et al. (2014)'s fuzzy arithmetic operation and a fuzzy version of simplex algorithm is proposed for solving fuzzy linear programming problem whose parameters all are represented by symmetric trapezoidal fuzzy number without converting the given problem into crisp equivalent problem. By using the proposed method the fuzzy optimal solution of fully fuzzy linear programming problem can be easily obtained. To illustrate the proposed method a numerical example has been solved. The proposed method can be extended to proposed Big-M and two-phase method for finding fuzzy optimal solution of fully fuzzy linear programming problems.

\section{Acknowledgement}

The authors would like to thank the anonymous referees for constructive comments on earlier version of this paper.

\section{References}

Allahviranloo, T., Lotfi, F. H., Kiasary, M. K., Kiani, N. A., \& Alizadeh, L. (2008). Solving fully fuzzy linear programming problem by the ranking function. Applied Mathematical Sciences, 2(1), 19-32.

Bellman, R. E., \& Zadeh, L. A. (1970). Decision-making in a fuzzy environment. Management science, 17(4), B-141.

Bhardwaj, B., \& Kumar, A. (2013). A Note on the Paper "A Simplified Novel Technique for Solving Fully Fuzzy Linear Programming Problems". Journal of Optimization Theory and Applications, $163,1-12$.

Buckley, J. J., \& Feuring, T. (2000). Evolutionary algorithm solution to fuzzy problems: fuzzy linear programming. Fuzzy Sets and Systems, 109(1), 35-53.

Dehghan, M., Hashemi, B., \& Ghatee, M. (2006). Computational methods for solving fully fuzzy linear systems. Applied Mathematics and Computation,179(1), 328-343.

Ezzati, R., Khorram, E., \& Enayati, R. (2014). A particular simplex algorithm to solve fuzzy lexicographic multi-objective linear programming problems and their sensitivity analysis on the priority of the fuzzy objective functions. Journal of Intelligent and Fuzzy Systems, 26(5), 2333-2358.

Ganesan, K., \& Veeramani, P. (2006). Fuzzy linear programs with trapezoidal fuzzy numbers. Annals of Operations Research, 143(1), 305-315.

Hashemi, S. M., Modarres, M., Naserabadi, E. \& Naserabadi, M. M. (2006). Fully fuzzied linear programming, Solution and duality. Journal of Intelligence Fuzzy Systems, 17, 253-261.

Hatami, A., \& Kazemipoor, H. (2014). Solving fully fuzzy linear programming with symmetric trapezoidal fuzzy numbers using Mehar's method. Journal of Mathematical and Computational Science, 4(2), 463-470.

Hosseinzadeh Lotfi, F., Allahviranloo, T., Alimardani Jondabeh, M., \& Alizadeh, L. (2009). Solving a full fuzzy linear programming using lexicography method and fuzzy approximate solution. Applied Mathematical Modelling, 33(7), 3151-3156.

Khan, I. U., Ahmad, T., \& Maan, N. (2013). A simplified novel technique for solving fully fuzzy linear programming problems. Journal of Optimization Theory and Applications, 159(2), 536-546.

Kumar, A., Kaur, J., \& Singh, P. (2010). Fuzzy Optimal Solution of Fully Fuzzy Linear Programming Problems with Inequality Constraints. International Journal of Applied Mathematics \& Computer Sciences, 6(1), 37-41.

Nasseri, S. H., Behmanesh, E., Taleshian, F., Abdolalipoor, M., \& TaghiNezhad, N. A. (2013). Fully fuzzy linear programming with inequality constraints. International Journal of Industrial Mathematics, 5(4), 309-316.

Tanaka, H., Okuda, T., \& Asai, K. (1974).On fuzzy mathematical programing. The Journal of 
124

Cybernetics, 3, 37-46.

Zimmermann, H.J. (1978). Fuzzy programming and linear programming with several objective functions. Fuzzy Sets and Systems, 1, 45-55. 